\title{
The Nonvelocity Cosmological Spectral Shift
}

\author{
Paul A. LaViolette \\ The Starburst Foundation, Niskayuna, NY, USA \\ Email: plaviolette@starburstfound.org
}

How to cite this paper: LaViolette, P.A. (2021) The Nonvelocity Cosmological Spectral Shift. International Journal of Astronomy and Astrophysics, 11, 232-251. https://doi.org/10.4236/ijaa.2021.112012

Received: March 27, 2021

Accepted: May 28, 2021

Published: June 1, 2021

Copyright $\odot 2021$ by author(s) and Scientific Research Publishing Inc. This work is licensed under the Creative Commons Attribution International License (CC BY 4.0).

http://creativecommons.org/licenses/by/4.0/

\begin{abstract}
Beginning from the premise that the universe is static, and that the cosmological redshift is due to a nonconservative tired light effect, the following examines evidence supporting the prediction that photons will progressively blueshift when transiting through the gravity wells of galaxies, galaxy clusters, and superclusters. The presence of such a nonvelocity blueshift effect is seen to make a substantial contribution to Virgo cluster galaxy spectra, sufficient to dramatically decrease the cluster's velocity dispersion and assessed virial mass and eliminate the need to assume the presence of large quantities of dark matter. The effect is also shown to account for the Fingers-of-God effect and Kaiser pancaking effect seen when the spectra of cluster galaxies are plotted in redshift space. The opposite effect, excessive redshifting of photons passing through cosmic voids is able to explain void elongation in redshift space, and also the subnormal luminosity of void galaxies. The proposed cosmological blueshifting phenomenon also explains the downturn of the slope of the Hubble Flow in the vicinity of the Local Group which projects a negative apparent velocity for photons propagating near the Milky Way. It also offers an explanation for the blueshift of the Andromeda galaxy spectra and for Arp's findings that the spectra of primary galaxies in a cluster tend to be blueshifted relative to their companion galaxies. These photon energy phenomena are anticipated by the physics of subquantum kinetics which predicts that photons traversing long distances through intergalactic space should undergo nonconservative tired-light redshifting, and that photons passing through gravity potential wells should undergo progressive blueshifting. The latter effect may be visualized as a negative nonvelocity Hubble constant.
\end{abstract}

\section{Keywords}

Fingers-of-God Effect, Kaiser Effect, Virgo Cluster, Dark Matter, Cosmic Voids, Hubble Flow, Local Group, Hubble Constant, Nonvelocity Spectral Shifts, Cosmological Redshift, Subquantum Kinetics 


\section{Introduction}

There are a number of astronomical phenomena which have currently posed a challenge to astronomy, such as the Virgo cluster blueshift, the Fingers-of-God effect, the Kaiser effect, the blueshift bias of the Local Group relative to the Hubble flow, and the observation that primary galaxies in a cluster tend to be blueshifted relative to the spectra of their companion galaxies. This paper intends to show that all of these effects can be accounted for if one allows a small violation in energy conservation and assumes that a photon's wavelength progressively blueshifts as the photon traverses regions of space where the gravitational potential is particularly negative, as when passing through a galaxy cluster gravity well. The amount of violation required is extremely small, roughly 10 orders of magnitude smaller than what can be verified over laboratory distances. Hence it is reasonable to explore such a possibility, and as is shown below, this phenomenon does have a sound theoretical basis.

For most of their history, physics and astronomy have operated on the assumption that the universe behaves as a closed system and that the First Law applies rigorously to all physical phenomena. However, this world view has become strained by recent observations of supermassive galactic cores in an active state of eruption which lack dust in their immediate vicinity to fuel their enormous energy output. Moreover, as Arp [1] has shown, such cores may birth and expel entire galactic embryos, raising concerns about how this is possible without violating energy conservation.

Another serious threat to the closed system paradigm is posed by the imminent collapse of the big bang cosmology. The big bang theory side-stepped its immense energy nonconservation violation by claiming that its matter/energy creation event occurred in a time shorter than the Planck time. However, as galaxies have been observed at increasingly large redshifts in recent decades, evidence has mounted against the expansion hypothesis as the static universe, tired light model has been found to make a far better fit to cosmological test data. In particular, the superiority of the static universe, tired light model is best seen when the competing cosmologies are simultaneously compared on multiple cosmology tests [2] [3]. These findings raise the question as to what creation process should replace the big bang. The natural replacement cosmology would be one that predicts continuous matter creation [3]. But since such creation would necessarily span tens or hundreds of billions of years, there is no easy way of avoiding the possibility that the First Law would be continuously broken with the emergence of each new particle of matter.

As is well known, the field of nonequilibrium thermodynamics teaches that energy is not necessarily conserved in open systems. Hence, puzzles posed by supermassive active galactic cores, as well as the downfall of the big bang theory and its imminent replacement with continuous creation alternatives, should herald in a new era in which the universe is instead conceived to operate as an open system. One such open system approach that is considered here is that of 
the subquantum kinetics (SQK) physics methodology [4] [5] [6] [7] [8]. This draws concepts from the field of chemical kinetics and applies them to microphysics as a description of the subquantum domain. The theoretical approach of SQK is very different than that of contemporary physics. Hence the reader is advised to consult the above references to get a better understanding of its basis.

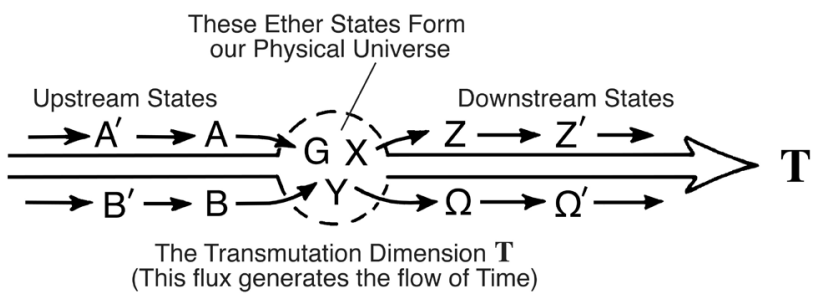

Figure 1. The subquantum kinetics ether reaction scheme as it would appear disposed along transformation dimension T. G, X, and Y mark the domain of the physical universe.

Nevertheless, at the risk of confusing the reader who may be unfamiliar with this approach, a brief summary is provided below.

According to this view, all space is assumed to be pervaded by a subquantum reaction-diffusion medium, or ether, made up of different types of particulate subquantum entities called "etherons". Furthermore, SQK postulates that the physical universe at the subquantum level is inherently processual, with etherons entering our universe by transforming from "upstream" states and leaving our universe by transforming into "downstream" states as shown in Figure 1. Our entire universe is then postulated to occupy a point along a higher dimensional vector of unidirectional transformation $\mathrm{T}$ which defines an unobservable flux that essentially passes "through" our universe at the subquantum level. This flux is postulated to sustain and animate all quantum phenomena. How this proposed subquantum flux may be related to our experience of the flow of Time has been discussed in previous publications [7] [9]. It should be noted that this idea is not new. Similar descriptions of a processual ether appear in nineteenth century ether theories [10] as well as in ancient cosmologies such as that of Heraclitus, the I Ching, and Hindu scripture [9].

In the SQK scheme, etherons are postulated to react with one another or transform one into another in a specific manner as described by a set of five kinetic equations called Model G (Equation system (1)):

$$
\begin{gathered}
\mathrm{A} \underset{k_{-1}}{\stackrel{k_{1}}{\rightleftarrows}} \mathrm{G} \\
\mathrm{G} \underset{k_{-2}}{\stackrel{k_{2}}{\rightleftarrows}} \mathrm{X} \\
\mathrm{B}+\mathrm{X} \underset{k_{-3}}{\stackrel{k_{3}}{\rightleftarrows}} \mathrm{Y}+\mathrm{Z} \\
\mathrm{X}+\mathrm{Y} \underset{k_{-4}}{\stackrel{k_{4}}{\rightleftarrows}} \mathrm{X} \\
\mathrm{X} \underset{k_{-5}}{\stackrel{k_{5}}{\rightleftarrows}} \Omega
\end{gathered}
$$


The letters represent the concentrations of various ether reactants, and the arrows indicate their direction of transformation at rates specified by kinetic constants $k_{i}$. In all cases, the forward reactions are assumed to be dominant. The concentrations of $\mathrm{A}$ and $\mathrm{B}$, the source reactants, and $\mathrm{Z}$ and $\Omega$, the sink reactants, are assumed to remain constant, while $\mathrm{G}, \mathrm{X}$, and $\mathrm{Y}$ are allowed to vary over space and time. In addition, each reactant is associated with a diffusion coefficient such that, in its entirety, Model G constitutes a reaction-diffusion system similar in some respects to the well-known Brusselator reaction system. Variations in space and time of $\mathrm{G}$ would be observable at the quantum level as gravitational potential field variations, $\varphi_{g}(r, t)$, and variations of $\mathrm{Y}$ would correlate with variations in the electric potential field, $\varphi_{y}(r, t)$. The $\mathrm{X}$ variable also plays an important role with $\mathrm{Y}$ in generating the electric field's bipolar aspect.

In this scheme, the $G$ variable, and hence gravity potential, serves as the bifurcation parameter that determines whether this nonlinear reaction system operates in a subcritical or supercritical mode. The astrophysicist unfamiliar with SQK, might be helped by imagining a given volume of space as functioning like the core of a nuclear reactor where the ambient gravity potential acts like the reactor control rods regulating the criticality of the nuclear reaction and determining whether the underlying reaction system operates in a subcritical, energy damping mode, or in a supercritical energy amplifying mode. Nonlinear reaction-diffusion systems in general exhibit similar modes of nonconservative wave behavior, for example, see Gmitro and Scriven [11].

When the concentration of $G$ is above a certain critical threshold $G_{0}$, that is when $\varphi_{g}>0$, Model G becomes subcritical. Energy potential fluctuations from the steady state will then regress in amplitude. Alternatively, a photon's energy will exponentially decrease as a function of travel distance as follows:

$$
E(r)=E_{0} \mathrm{e}^{-\left(\alpha \varphi_{g} / c\right) r}
$$

where $E_{0}$ represents the photon's initial energy, $E(r)$ signifies its energy after traveling a distance $r$ from its point of origin, $\alpha$ is a constant of proportionality given in units of $\mathrm{s} / \mathrm{cm}^{2}, \varphi_{g}(r)$ is the ambient gravity potential in the region traversed by the photon given in units of $\mathrm{cm}^{2} / \mathrm{s}^{2}$, and $c$ is the velocity of light in $\mathrm{cm} / \mathrm{s}$. This nonconservative energy expression follows as a consequence of the behavior of the Model G reaction system [6] [7]. It does not violate the First Law since SQK conceives the Universe to function as an open system, the photon's field potentials being sustained by the underlying reaction-diffusion processes.

When the concentration of $\mathrm{G}$ is below its critical threshold $\mathrm{G}_{\mathrm{o}}$, or when $\varphi_{g}<0$, Model G instead becomes supercritical and energy potential fluctuations from the steady state spontaneously increase in amplitude. In this case, the exponent in Equation (2) becomes positive, and photons traveling through regions of negative gravity potential are predicted to exponentially increase their energy with travel distance. When $\varphi_{g}=0$, the system operates at its threshold of marginal stability, and the exponent in Equation (2) becomes unity dictating perfect energy conservation. 
The various modes of photon energy behavior predicted by this physics are illustrated in Figure 2. In the SQK paradigm, gravity potential fields are predicted to have a finite extent similar to modified Newtonian dynamics or MOND. Hence, at the center of a galaxy or cluster, gravity potential is negative and progressively rises with increasing distance from this center, eventually tapering off to a local positive intergalactic value. This tapering and limited extent of the gravity field is advantageous for static universe cosmologies in general and specifically for that of SQK since there is no tendency for the induction of a spontaneous cosmic collapse.

Defining the coefficient $\beta(r)=\alpha \varphi_{g}(r) / c$, Equation (2) becomes expressed as:

$$
E(r)=E_{0} \mathrm{e}^{-\beta r}
$$

which dictates nonconservative energy attenuation for $\beta>0$, and nonconservative energy amplification for $\beta<0$. Expressed in terms of photon wavelength, $\lambda$, Equation (3) may alternatively be written as:

$$
\lambda(r)=\lambda_{0} \mathrm{e}^{\beta r}
$$

There is substantial evidence that the cosmological redshift is quantized and changes in increments that are multiples of $12 / \mathrm{km} / \mathrm{s}$, and the same likely occurs for the cosmological blueshift effect. Hence the exponential term in Equation (4) may be written in the form $n \beta \Delta r$, or alternatively as $n \Delta z$ [2] [3].

Since a photon from a distant galaxy would spend far more time traveling through subcritical intergalactic void regions than through supercritical regions surrounding galaxy clusters, its wavelength on average is predicted to progressively increase as it travels over cosmological distances. In this averaged case, with $\beta>0$, relation (4) describes progressive photon redshifting identical with the tired-light relation first proposed by Zwicky [12] to explain Hubble's redshift-distance observations. As mentioned above, studies checking competing cosmologies against data on multiple cosmology tests [2] [3], as well as studies referenced therein, show that the data favors the static universe, tired-light relation over the no evolution expanding universe hypothesis. In particular, the tired light effect predicted by SQK does not require that the photon undergoes

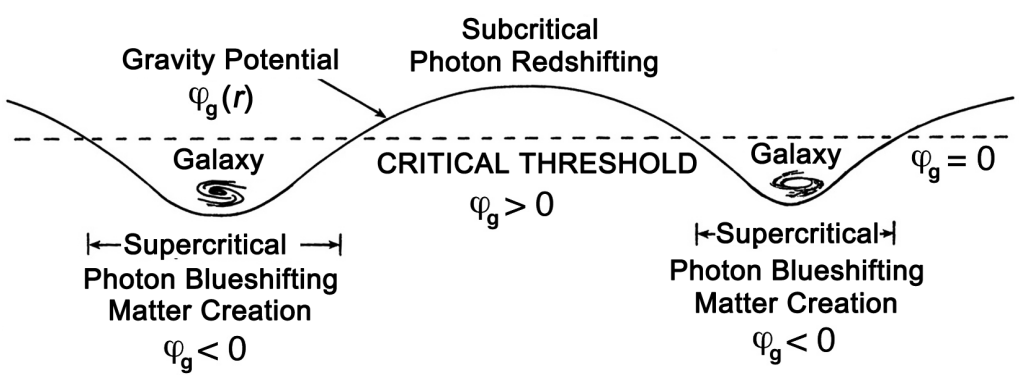

Figure 2. According to SQK, a photon's energy behavior depends on the ambient value of the gravity potential in the photon's vicinity judged relative to the critical threshold value, $\varphi_{g}=0$. Photons are predicted to progressively increase their energy within the gravity wells that surround galaxies and galaxy clusters (where $\varphi_{g}(r)<0$ ), and to progressively decrease their energy in intergalactic space (where $\varphi_{g}(r)>0$ ). 
scattering interactions with its environment. Hence redshifting occurs in the same fashion at both visible and radio frequencies and also parent galaxy image blurring should not be seen, thereby circumventing criticisms often made of other tired-light models.

To be physically realistic, the specific values for $\alpha$ and $\varphi_{g}$ in Equation (2) must be chosen to match observation. For example, in the case of energy damping occurring over cosmological distances, this would be similar to ensuring that photons lose energy at a rate that is consistent with the observed Hubble constant value. Similarly, standard expanding universe cosmological theory does not predict a specific value for the Hubble constant, but adopts a value based on astronomical observation. Attenuation coefficient $\beta$ may be expressed in terms of the Hubble constant as $\beta=H_{0} / c$. Adopting a value of $H_{0}=64.0 \pm 1.6 \mathrm{~km} / \mathrm{s} / \mathrm{Mpc}$, as proposed by Tammann and Reindl [13], would give a $\beta$ value $6.54 \pm 0.16 \%$ per billion light years, or $2.13 \pm 0.05 \times 10^{-4} / \mathrm{Mpc}$. It should be kept in mind that we use the Hubble constant symbol here in a nonvelocity context, rather than in the expansion context as is more commonly employed. Why this value is chosen, as opposed to other values published for $H_{0}$ is described in the related paper by LaViolette [3] which notes that choosing an $H_{0}$ close to this value allows the tired-light model to make a good fit to high-redshift Hubble diagram plots of supernova and gamma ray burst data in studies carried out by Marosi [14] [15]. When this attenuation is expressed in terms of time rather than distance, relation (4) becomes:

$$
\lambda(r)=\lambda_{0} \mathrm{e}^{\mu t}
$$

where $\mu=\beta c=2.07 \pm 0.05 \times 10^{-18} / \mathrm{s}$.

This gravity-dependent tired-light redshift effect is unique to SQK and has nothing to do with the more widely known gravitational redshift of the general theory of relativity. Although, SQK does account for conventional relativistic effects as well [5] [7] [16]. Regardless of the specific values chosen for $\beta$, or $\mu$, whether they be positive values, denoting exponential energy decrease, or negative values, denoting exponential energy increase as occurs during passage through a gravity potential well, the overall character of these relations is exponential.

The focus of the present paper is to explore evidence that the Hubble "constant" may actually be variable, namely, to seek evidence for the prediction that nonvelocity photon blueshifting occurs in galaxy clusters and superclusters or that excessive redshifting occurs when photons pass through cosmic voids. It is found that a variable Hubble constant is able to explain a variety of astrophysical phenomena which for some time have been puzzling when understood from the perspective of standard cosmology.

\section{Nonvelocity Photon Blueshifting When Transiting the Virgo Cluster}

The Virgo cluster lies at a distance of about $16.5 \mathrm{Mpc}$ and is the largest galaxy 
cluster within $100 \mathrm{Mpc}$ of our Local Group (LG). Figure 3 plots the spectral shifts of galaxies observed in and near the Virgo cluster as a function of distance for a line-of-sight directed through the cluster's center. The data for this is taken from Figure 1 of the paper by Mei et al. [17] who plot their galaxy spectral shift data transformed from the heliocentric rest frame of the observer to the cosmic microwave background rest frame with the assumption that the Milky Way has a moderate relative velocity. The redshift-distance trend lines seen here, solid lines (a) and (b) are least squares fits made for galaxies lying outside of the immediate vicinity of the Virgo cluster, and are adapted from the paper of Tonry, et al. [18]. Their data are here plotted to show how the apparent relative velocity of a photon changes as it journeys towards Earth from an arbitrary distance of $40.5 \mathrm{Mpc}$ where the photon's spectral shift is arbitrarily set to zero for the purpose of illustration.

Viewed along our line of sight, the redshift-distance trend lines for galaxies located both behind the cluster (line a) and in front of the cluster (line b) are reported to conform to a Hubble constant of approximately $78 \mathrm{~km} / \mathrm{s} / \mathrm{Mpc}$. These two trend lines are seen to be displaced vertically relative to one another as if photons journeying from galaxies on the far side of the cluster had been collectively blueshifted as they passed through the cluster. This presents an insurmountable difficulty for interpretations that attempt to explain the anomalous redshifts and blueshifts seen in the vicinity of the Virgo cluster as being due to the peculiar velocities of galaxies relative to our line of sight. For, if these galaxies were moving in symmetric fashion around Virgo's center of mass, one would expect an equal proportion of redshifted and blueshifted galaxies. Instead, we see that galaxies whose line-of-sight position lies close to the cluster center have an imposed blueshift bias. Neither can this discrepant blueshift be realistically explained by an assumed motion of the Local Group toward Virgo. So, the observed blueshift offset remains unexplained by standard theory.

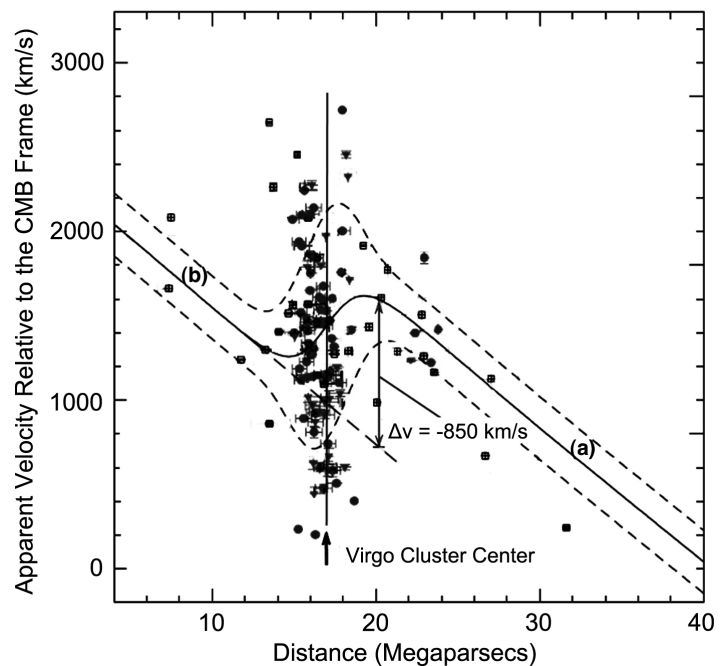

Figure 3. Tired-light redshifting and temporary blueshifting experienced by distant photons as they traverse through the Virgo cluster. 
According to the nonvelocity interpretation presented here, suppose that a photon begins its journey toward us from a distance of $40.5 \mathrm{Mpc}$, progressively losing energy with increasing travel distance, and redshifting at a rate of $H_{0}=$ $+78 \mathrm{~km} / \mathrm{s} / \mathrm{Mpc}$, trend line (a). Upon nearing the Virgo cluster, and beginning around 3.7 Mpc from the cluster's center, prevailing supercritical conditions cause this redshifting trend to reverse and turn into a blueshifting trend, depicted by the sigmoid line joining the two z-r trend lines. The redshift-distance relation within the cluster now yields a downward slope of roughly -120 $\mathrm{km} / \mathrm{s} / \mathrm{Mpc}$, see Figure 2. Once the photon has passed through the cluster and reached the side of the cluster facing the Milky Way, this blueshifting trend begins to taper off and to finally reverse into a redshifting trend once again, trend line (b). The redshift-distance relation on the near side of the Virgo cluster here converges to the same value it exhibited on the far side: $+78 \mathrm{~km} / \mathrm{s} / \mathrm{Mpc}$.

Trend line (b) is displaced downward relative to trend-line (a) by an apparent velocity of $-850 \mathrm{~km} / \mathrm{s}$. Hence photons appear to blueshift at the rate of -120 $\mathrm{km} / \mathrm{s} / \mathrm{Mpc}$ over a central cluster distance of approximately $7 \mathrm{Mpc}$, acquiring a total energy gain 0.28 percent, or $(-850 \mathrm{~km} / \mathrm{s}) / \mathrm{c}$, in the course of their passage through the cluster. This translates into a frequency blueshifting rate of $\mu=-3.9$ $\times 10^{-18} / \mathrm{s}$ which is about 2.5 times the photon blueshifting rate that SQK predicts to occur in the Earth's vicinity [7]. It is also about twice the rate of energy change that photons encounter on their cosmological journey, but of opposite sign.

Tonry, et al. [18] refer to this sigmoid apparent velocity feature as Virgo's "s-wave". Alternatively, they refer to it as the s-wave distortion of the Hubble flow, interpreting the spectra in terms of the velocity paradigm. Their interpretation postulates positive peculiar velocities being present on the near side and negative peculiar velocities being present on the far side of Virgo. However, in the new paradigm being proposed here, the characteristic S-shape of Virgo's redshift-distance relationship is not due to coordinated peculiar motions of galaxies, but rather is evidence that the Virgo cluster resides in an energy-amplifying supercritical region.

Note that the value of $H_{0}=78 \mathrm{~km} / \mathrm{s} / \mathrm{Mpc}$ found locally in front of and behind the Virgo cluster is much larger than the value $H_{0}=64 \mathrm{~km} / \mathrm{s} / \mathrm{Mpc}$ adopted for cosmological distances. However, this value drops considerably when the Virgo cluster blueshifting interval is included. For example, referring to Figure 2, photons traveling from a distance of $40 \mathrm{Mpc}$ to $4 \mathrm{Mpc}$ increase their apparent velocity redshift by $2000 \mathrm{~km} / \mathrm{s}$, hence giving an average Hubble redshift rate of $56 \mathrm{~km} / \mathrm{s} / \mathrm{Mpc}$. However, considering an even larger span of distance behind the Virgo cluster and assuming that the $78 \mathrm{~km} / \mathrm{s} / \mathrm{Mpc}$ trend line continues to hold, the resulting average value obtained for $H_{0}$ would accordingly increase and approach closer to the cosmological value. So, it seems reasonable that when cosmological distances are considered which would accompany the passage of photons through successive galaxy cluster gravity wells and cosmic void gravity hills, that the average value obtained for this Hubble spectral shift parameter would be 
even greater, converging to the above cited $H_{0}$ value. Perhaps the Hubble spectral shift parameter is better represented as $H_{\varphi}$ to denote that it is actually a gravity potential dependent variable when considering comparatively small distance spans.

In the SQK cosmology, each galaxy cluster resides within a supercritical gravity well, whose gravity potential is theorized to fall below the critical threshold value $\varphi_{g}=0$. As described elsewhere [3] [4] [5] [6] [7], SQK predicts that such "fertile" cluster gravity wells are sites where photon energy is continually amplified, and matter is continuously created. It proposes that such supercritical regions may have existed in primordial times even before the emergence of matter, and that the reason why matter abounds in these regions today is because of the supercritical, energy-amplifying conditions that exist there. In primordial times, such fertile pockets could have been created by quasi-periodic multi-megaparsec steady-state spatial variations in the concentrations of certain of Model G's reactants [7]. SQK predicts that zero-point energy fluctuations would grow and eventually nucleate primordial neutrons in these pockets, which in turn would decay into protons and electrons, forming an ionized hydrogen gas cloud, each particle serving as a nucleation center for further matter proliferation through a process of continuous creation. Once cool enough, molecular hydrogen would form and coalesce to form planetesimals, which would eventually grow into stars which with further proliferation would form primordial galaxies [3] [6] [7]. The more massive the galaxy, the deeper and more extensive would be its gravity well. Once a number of galaxies form in a given primordial gravity well, their respective gravity wells would act together to collectively maintain the cluster's gravity well, locally lowering its collective $\varphi_{g}(r)$ potential. Dark matter is not necessary to explain the presence of these clusters. Note that due to the limited reach of galactic gravity fields in SQK, such aggregations would not have formed through gravitational coalescence.

In speaking about photon blueshifting in galactic clusters, it is important also to consider the effect of the more conventional gravitational redshift effect. For example, a star in a galaxy in the Virgo cluster would likely have a gravity potential that is much more negative than Earth's. Hence photons arriving from that galaxy will necessarily undergo a gravitational redshift that would subtract from any cosmological photon blueshifting they underwent on their journey to us. Cappi [19] has estimated the gravitational redshift of galaxy clusters by assuming a de Vaucouleurs profile. For example, he finds that a cluster that has a galaxy apparent velocity dispersion of $\sim 1000 \mathrm{~km} / \mathrm{s}$ would have a gravitational redshift of $\sim 80 \mathrm{~km} / \mathrm{s}$. Also, Stiavelli and Setti [20] have studied the radial velocity profiles of 24 individual galaxies to estimate their gravitational redshifts. As an example, two giant elliptical galaxies in the Pegasus constellation, NGC 7626 and NGC 7619 , believed to have masses of $2 \times 10^{12} \mathrm{M}_{\odot}$ and $10^{12} \mathrm{M}_{\odot}$ respectively, are estimated to have gravitational redshifts of $16 \mathrm{~km} / \mathrm{s}$ and $7 \mathrm{~km} / \mathrm{s}$ respectively. But these various redshift values are small in comparison to the overall blueshifting 
observed for the spectra of these clusters, and for the purposes of the present discussion may be ignored.

\section{The Fingers-of-God Effect and the Kaiser Effect}

When the spectral shifts of cluster galaxies are mapped out in redshift space to form a wedge diagram such as that shown in Figure 4 for the Virgo cluster [21], they are found to adopt an elongated, appendage-like distribution directed towards the observer's location. This same phenomenon is also seen when other clusters as well are plotted in redshift space. In each case, a blueshifted spectral "finger" appears to point towards Earth, as if by divine plan, a phenomenon termed the Fingers-of-God effect.

The standard explanation is that the galaxies in the cluster have random peculiar motions with velocity components being directed both towards and away from the observer. In the case of the Virgo cluster, if three high redshift galaxies are excluded, it is found that the galaxy spectra span a range $\Delta \mathrm{v}=3400 \mathrm{~km} / \mathrm{s}$; see Figure 6 of Arp's paper [21]. This spectral spread is much greater than that shown in Figure 4, which does not plot blueshifted Virgo cluster galaxies from 0 to $-350 \mathrm{~km} / \mathrm{s}$. If all of Virgo's spectral spread is inferred to be due to peculiar velocities of galaxies on the order of $\pm 1700 \mathrm{~km} / \mathrm{s}$, gravitational attraction due to visible matter is found to be insufficient to hold the cluster together. To retain the assumption that these spectral shifts are due to peculiar velocities and to avoid the ultimate kinetic dispersal of the galaxies, the assumption is usually made that the cluster contains large amounts of dark matter. But since there is no way to independently verify whether dark matter really exists, this is essentially an ad hoc assumption.

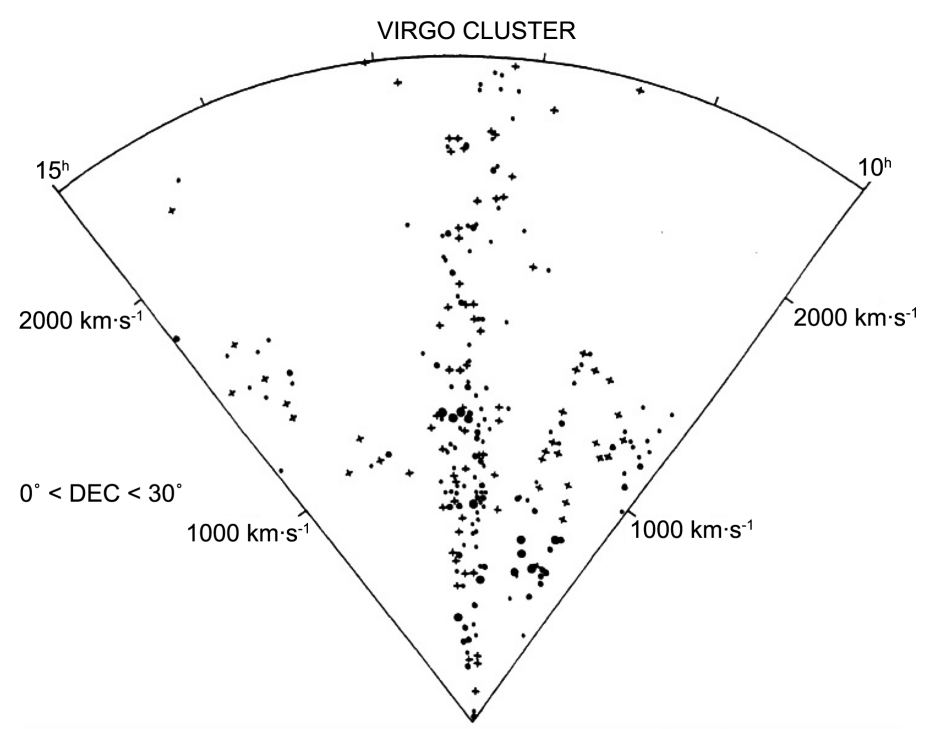

Figure 4. Wedge diagram for the Virgo cluster (after H. Arp [21]). Crosses represent spiral galaxies Sc or later. Symbol size is proportional to apparent magnitude. Note the long vertical distribution of galaxies in redshift space. Galaxies situated further from the line of sight, hence toward the edge of the cluster's gravity well. 
The dark matter assumption may be partially or entirely eliminated when it is realized that a major portion of Virgo's spectral shift spread may be due to nonvelocity blueshifting. For the purpose of illustration, suppose that $850 \mathrm{~km} / \mathrm{s}$ of Virgo's spectral spread is due to the nonvelocity blueshift acquired by photons passing through the Virgo cluster supercritical region, as shown in Figure 3. This then would leave $2550 \mathrm{~km} / \mathrm{s}$ which could be attributed to cluster kinematics, i.e., to galaxies having peculiar motions of about $\pm 1275 \mathrm{~km} / \mathrm{s}$. This is about $75 \%$ of Virgo's velocity spread and if this reduced amount were attributed to peculiar velocity, the dynamical mass estimated for Virgo would be reduced to $56 \%$ of its current value.

But in making this estimate we have not taken into account the blueshifting that photons experience as they pass through the gravity well of the galaxy from which they originate. Arp [21] finds that the most massive and luminous galaxies in the Virgo cluster, that is galaxies with deep gravity wells, are usually the ones with the greatest blueshifts. He also finds that Virgo's less luminous galaxies as well as its type-Sc galaxies, that is, those having relatively shallow gravity wells, are the ones with the greatest redshifts. This is exactly in accordance with the predicted gravity potential dependent blueshifting effect. Arp does not quantify the contribution of this effect relative to Virgo's spectral spread, but judging from Figure 6 in his paper, it is reasonable to suppose that it accounts for at least $400 \mathrm{~km} / \mathrm{s}$ of the total spread. Subtracting this we find that Virgo's peculiar velocity spread now reduces to $2150 \mathrm{~km} / \mathrm{s}$, implying velocities of $\pm 1075 \mathrm{~km} / \mathrm{s}$, which is about $63 \%$ of the observed amount. This then implies that Virgo's dynamical mass is $40 \%$ of its currently assumed amount, greatly reducing the need to assume the presence of large amounts of dark matter. Additionally, if one considers that the sloping outer wall of Virgo's gravity well would exert a centripetal force on galaxies within its cluster helping to shepherd its galaxies inward, the need for dark matter would be further reduced and possibly even entirely eliminated.

The standard assumption that the excessive galaxy blueshifts in clusters are due to galaxy kinematics runs into even more trouble when larger more distant clusters are considered. For example, the wedge diagram for the Shapley supercluster shows galaxy redshifts extending from less than $1000 \mathrm{~km} / \mathrm{s}$ out to 60,000 $\mathrm{km} / \mathrm{s}$. This velocity spread is so large that attempts to introduce assumptions about the presence of dark matter lead to absurdities. Standard cosmology is then forced to assume that these redshifts are mainly cosmological in nature and that the cluster is shaped like an immense sausage whose axis is oriented along our line-of-sight to form a "Fingers-of-God" in real space (not just in redshift space). Suggestions that this orientation is due to chance are inadequate since this supercluster is just one of many galaxy clusters that exhibit such pointing effects. Adopting a Hubble constant value of $H_{0}=64 \mathrm{~km} / \mathrm{s} / \mathrm{Mpc}$, this leads to the conclusion that the Shapley supercluster extends from the solar neighborhood out to a distance of $890 \mathrm{Mpc}$, with galaxies at the far end of the cluster receding from the cluster's low redshift galaxies at almost $20 \%$ c in the standard expan- 
sion hypothesis. Over such large distances, and such a large spread of velocities, clusters would be unable to keep from dispersing, even if dark matter and standard Newtonian gravity fields are assumed. The only way to avoid the problem is to admit that the redshifts of these galaxies are not entirely Doppler in nature, which causes fundamental problems for the expanding universe interpretation of cosmological redshifts.

As in the Virgo cluster, the SQK cosmological blueshifting effect offers a solution. It predicts that photons traveling towards us from galaxies either located within the cluster core or on the far side of the cluster will necessarily traverse the cluster's supercritical gravity well and be blueshifted to such an extent as to appear greatly displaced toward the observer in redshift space to produce a very elongated appendage-like distribution, as shown in Figure 5 (left). This blueshifting effect also explains why the Fingers-of-God redshift distribution is cusp shaped. That is, galaxies aligned with our line-of-sight to the cluster's center will be expected to exhibit the greatest blueshifted displacement in redshift space as is observed. On the other hand, photons coming to us from peripheral galaxies positioned on either side of the line-of-sight would be expected to undergo a lesser amount of blueshifting because their trajectories take them through the fringe of the cluster's gravity well where space is not as supercritical and the blueshifting rate is lower. Hence those galaxies will appear further away from us in redshift space.

Subquantum kinetics also predicts a redshift inversion effect since galaxies residing on the far side of the cluster will have their spectra blueshifted more than galaxies residing on the cluster's near side and hence the more distant galaxies would appear closer to us in redshift space. For example, blueshifted galaxies like M86 and M90, which have apparent velocities of around $-350 \mathrm{~km} / \mathrm{s}$, would actually lie on the far side of the cluster, their light traversing the entire Virgo cluster supercritical region. The Seyfert galaxy NGC 4388, which has one of the largest redshifts observed in the Virgo cluster, about $+2525 \mathrm{~km} / \mathrm{s}$, would actually be situated on the near side of the cluster, hence around $15 \mathrm{Mpc}$ away from us.

This blueshifting effect will also cause the cluster fringes to appear pancaked toward the observer in redshift space, adopting the shape of an oblate ellipsoid; see Figure 5 (left). Such pancaking is in fact observed and is referred to as the Kaiser effect [22]. Standard cosmology attributes the Kaiser effect to coherent peculiar motions of the cluster galaxies. Thus, whereas the Fingers-of-God effect is conventionally attributed to random peculiar motions, just a bit further out toward the periphery of the same cluster we are asked to believe that these peculiar motions now become coherent in such a way as to explain this pancaking effect. So, standard theory burdens itself with assumptions in order to explain observations.

\section{Cosmic Voids}

Subquantum kinetics predicts that cosmic voids also should exhibit a form of the Fingers-of-God effect, but in this case due to excessive redshifting. Voids would 


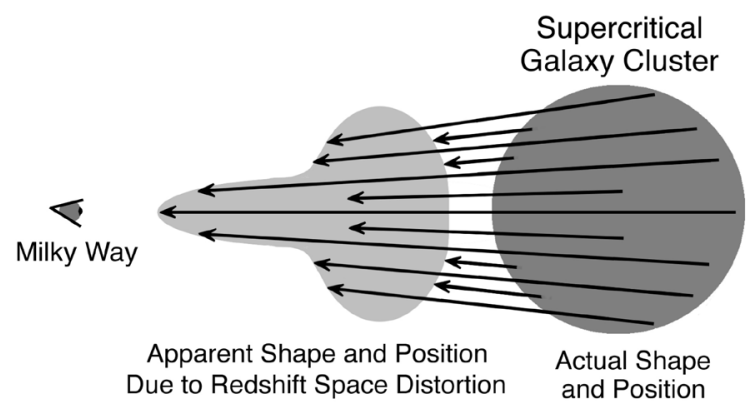

Figure 5. A typical galaxy cluster plotted in redshift space showing how the Fingers-of-God elongation effect and Kaiser pancaking effect are created when photons coming from the far side of an approximately spheroidal galaxy cluster undergo cosmological blueshifting during their passage through the cluster's supercritical region.

be regions which are more subcritical than surrounding regions of intergalactic space. Hence photons traveling to us through such voids would redshift (lose energy) more rapidly than the average cosmological rate, causing galaxies on the void's far side to appear to lie further away from us in redshift space. This would cause the voids to appear elongated along our line of sight as shown in Figure 6. In fact, void elongation is confirmed by observation. A study of the shapes of voids concludes that, when plotted in redshift space, they appear roughly as prolate ellipsoids with ellipticities averaging around 0.35 [23] [24]. That is, they appear about 1.5 times longer in the radial direction than in the transverse direction. Like the elongation effect observed in galaxy clusters, the voids give the impression that they are all "pointing" towards us.

The standard explanation is that this distortion arises because galaxies at the void boundaries have coherent peculiar velocities causing them to move as a group away from the void's center. Though, this creates a mystery as to what force standard theory would propose to be pulling them all away from these centers. Gravity itself is not strong enough unless one is willing to assume unreasonably large quantities of dark matter lurking beyond the void walls. The other suggestion that has been made to account for this phenomenon is to claim that the expanding universe deceleration parameter $q_{0}$ becomes negative inside voids, on the assumption, for example, that the void contains dark energy that causes it to expand more rapidly than surrounding regions of space [25]. However, such hypotheses are introduced in ad hoc fashion in order to explain how a spheroidal shaped void might appear elongated in redshift space. Subquantum kinetics, on the other hand, predicts this Fingers-of-God phenomenon without introducing additional assumptions.

Voids by definition are regions with fewer galaxies. According to SQK this has occurred because subatomic particles have had more difficulty nucleating in such regions because space is more subcritical there. Also, it predicts that matter creation should proceed far more slowly there. It is also worth noting that galaxies residing in cosmic voids are observed to be subluminous. Hoyle, et al. [26] have compared the luminosity distributions of distant void galaxies to distant 


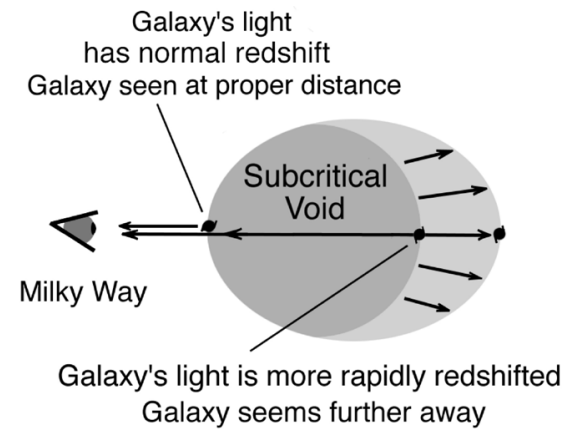

Figure 6. The apparent elongation of cosmic voids in redshift space as predicted by subquantum kinetics.

wall galaxies and have found that on average void galaxies are dimmer; see Figure 7. Murawski [27] has studied the void between the Virgo and Coma clusters and suggests that the dimming may be due to an intergalactic gas cloud causing $0.45 \mathrm{mag}$ of absorption. However, according to SQK, void galaxies would be dimmer because galaxies there would form gravity wells that are less supercritical than would galaxies in other regions of space. Hence the slower rate of photon blueshifting within the stars of these galaxies would result in stellar luminosities that would be lower when compared with galaxies located in a cosmic wall where the space is more supercritical. This finding mirrors the finding of Arp [21] that massive cluster galaxies are unusually luminous.

The consequence of the SQK photon blueshifting on the luminosities of planets, brown dwarfs, and stars of the lower and upper main sequence has been explored by the author elsewhere [7] [28].

The Local Void, the one that is closest to us, measures about $60 \mathrm{Mpc}$ in size and its nearest side lies about $2 \mathrm{Mpc}$ away from us, with the Local Group (LG) lying in the bordering cosmic wall. Some have suggested that the void is exerting a pressure on LG cluster galaxies, pushing them away toward the Virgo cluster which lies in the opposite part of the sky. Our galaxy, for example, is estimated to have an apparent relative velocity away from the void of about $260 \mathrm{~km} / \mathrm{s}$ [29]. Subquantum kinetics, on the other hand, suggests that much of the excess redshift normally attributed to peculiar galaxy recession could instead be an artifact of the excess nonvelocity redshifting that photons are undergoing as they journey to us through the void. In a similar fashion, if the nonvelocity photon blueshifting prediction is correct, the assumption that the Milky Way and local cluster galaxies are "falling" towards Virgo cluster, Great Attractor, or Shapley clusters due to excess blueshifting in those regions may need to be reevaluated.

\section{The Local Blueshift Bias}

According to SQK, photons should undergo cosmological blueshifting as they pass through the supercritical gravity well of our LG cluster. This is found to be the case. The redshift-distance regression line for dwarf galaxies, which is linear at distances greater than $2 \mathrm{Mpc}$, bends downward to negative apparent velocities at distances less than $2 \mathrm{Mpc}$, see Figure 8, after Karachentsev, et al. [30]. At 


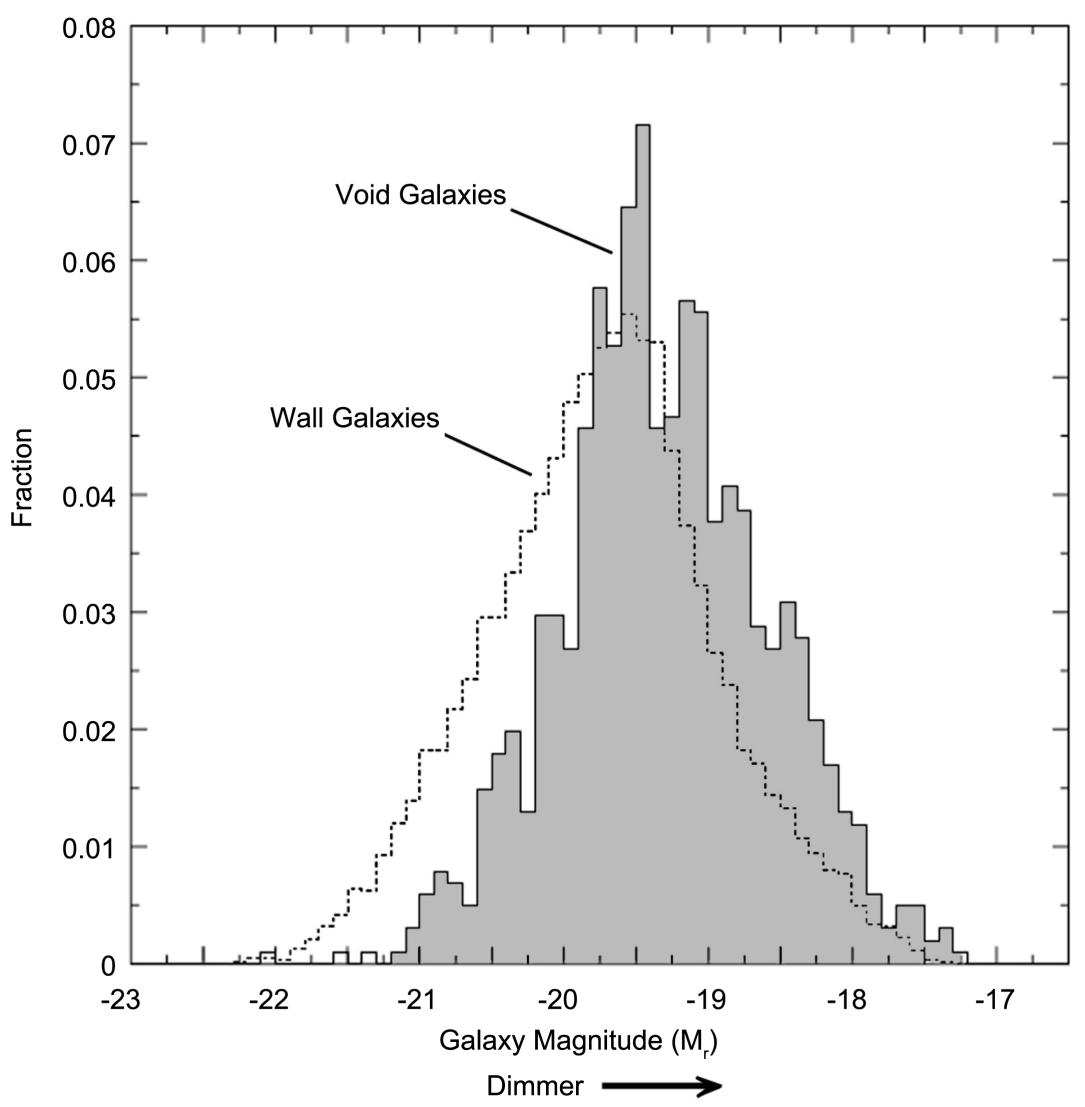

Figure 7. A comparison of the distribution of absolute magnitudes of void galaxies (shaded) to wall galaxies (after F. Hoyle, et al. [24]).

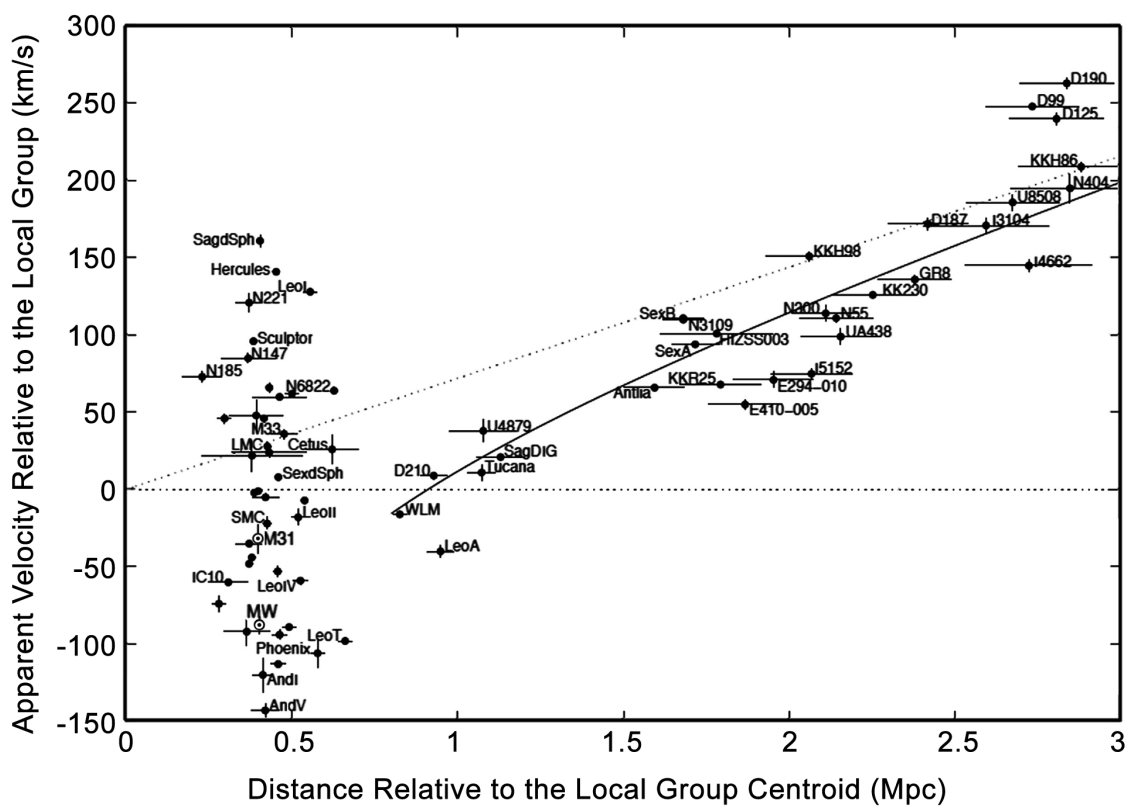

Figure 8. Spectral shifts (y axis) and distances (x axis) to various galaxies within $3 \mathrm{Mpc}$ of the Local Group, whose centroid is located at distance zero. The solid line plots the regression fit to the Hubble flow data. The horizontal dotted line marks where the apparent velocity attains a zero value. After Karachentsev, et al. [30]. 
about $3 \mathrm{Mpc}$ this regression line (solid line) exhibits a slope of $\sim 83 \mathrm{~km} / \mathrm{s} / \mathrm{Mpc}$ which progressively steepens to about $190 \mathrm{~km} / \mathrm{s} / \mathrm{Mpc}$ in the vicinity of the LG. It projects a negative spectral shift of $-75 \mathrm{~km} / \mathrm{s}$ at the location of the Milky Way (MW), which is stated to lie $0.43 \mathrm{Mpc}$ from the cluster centroid. The trend line, projects a zero redshift value at a distance of $\sim 1 \mathrm{Mpc}$ from the cluster barycenter. In the context of SQK, this suggests that the LG's supercritical region would have a radius of about $1 \mathrm{Mpc}$. This supercritical region would be far smaller than that of the Virgo cluster which likely has a radius of about $4 \mathrm{Mpc}$, based on Figure 3. Since the Virgo cluster is much more massive than the LG cluster, its gravity well would be much deeper, more supercritical, and much larger in extent, which would explain why it exhibits a far greater degree of photon blueshifting.

The conventional kinematic interpretation of Figure 8 is that the redshift-distance trend line in the vicinity of the LG, known as the Hubble flow, begins about $1 \mathrm{Mpc}$ from the LG centroid and that cosmological expansion is absent closer to the LG centroid. However, ad hoc assumptions must be added to the expansion hypothesis to account for it. In the SQK cosmological paradigm, on the other hand, this trend line dip is anticipated. Note that in the static universe tired-light interpretation of SQK, the term "Hubble flow" would be a misnomer in that galaxies would not actually be "flowing" away from us.

A related observation is that the Hubble flow is said to be very "cold", galaxies being observed to have a dispersion of only $\pm 2 \mathrm{~km} / \mathrm{s}$ around a $78 \mathrm{~km} / \mathrm{s} / \mathrm{Mpc}$ trend line. This has led Sandage [31] to comment "... the explanation of why the local expansion field is so noiseless remains a mystery." Also, Karachentsev, et al. [32] write, "The most enigmatic property of the local Hubble flow turns out to be its 'coldness'." However, this mystery evaporates when the local Hubble flow is understood to be due to a nonvelocity tired-light energy loss effect. The redshifting rate would be determined by the criticality of space which apparently does not vary much in our locale.

Subquantum kinetics also predicts that photons coming to us from the Andromeda galaxy should be blueshifted as they successively pass through Andromeda's supercritical gravity well, the LG cluster gravity well, and finally the Milky Way's gravity well. In fact, Andromeda is seen to exhibit a substantial blueshift. Arp and Sulentic [33] and Arp [1] estimate a value of $-86 \mathrm{~km} / \mathrm{s}$ based on the conclusion that the Sun has a Galactic orbital velocity of $220 \mathrm{~km} / \mathrm{s}$ [34]. Traditionally, Andromeda's blueshift has been interpreted kinematically as evidence that the galaxy has a velocity component directed toward the Milky Way. However, the findings of Arp [34] that the spectral shifts of Local Group companion galaxies relative to that of M31 are quantized in $72 \mathrm{~km} / \mathrm{s}$ steps with a standard deviation of $\pm 17 \mathrm{~km} / \mathrm{s}$ around those periodic values has led him to conclude that the peculiar velocities of our neighboring galaxies should be no greater than this $17 \mathrm{~km} / \mathrm{s}$. This is consistent with Arp's suggestion that M31's blueward spectral shift is for the most part nonvelocity in origin. The solution offered by SQK is that this blueshift is primarily due to local photon blueshifting. 


\section{Nonvelocity Blueshifting Influenced by Galaxy Mass and Morphology}

Arp [35] has studied galaxy groups consisting of a primary galaxy with interacting companions and has shown that companion galaxies have redshifts averaging $122 \pm 34 \mathrm{~km} / \mathrm{s}$ higher than that of the dominant galaxy, the more luminous and massive galaxy in the cluster. Regarding the spectral shift bias seen in the LG cluster, Arp and Sulentic [32] have stated that if the shifts are interpreted as peculiar velocities of the companions there is only one chance in 4 million that we would be viewing the cluster at a privileged time when all of its satellite galaxies happen to be collectively receding from us. Since 1970, at least 18 studies of this redshift anomaly have been carried out and all show the effect to be real. This has long been a thorn in the side of conventional astronomy since the concordance cosmology has no explanation for it. Bottinelli and Gouguenheim [36] have studied 20 galaxy groups containing 52 companion galaxies and confirmed that companion galaxies have redshifts that are systematically higher by about 90 $\pm 8 \mathrm{~km} / \mathrm{s}$. But they also considered the reverse, namely the lack of redshift in the main galaxy relative to the companion galaxies, and as expected found the main galaxy's redshift is generally lower by about $90 \mathrm{~km} / \mathrm{s}$, hence blueshifted relative to the companion galaxy spectra. This effect is predicted by the SQK blueshifting prediction. Namely, the more massive galaxies, such as giant ellipticals, should have deeper gravity wells and hence should undergo greater photon blueshifting than companion galaxies which are less massive and have shallower gravity wells. Also, primary galaxies are usually found at the center of a cluster, hence in a region of more negative gravity potential, where their spectra would be blueshifted to a much greater extent.

Arp [19] [37] and Russell [38] also report the presence of a redshift discrepancy that depends on galaxy morphology. They find that Sc and Scd "late type" galaxies have an excess redshift when compared to earlier type companion galaxies of Sa and Sb morphology. Part of this morphology-dependent redshift effect is anticipated by SQK. Sc and Scd spirals, for example, have arms that are more loosely wrapped and hence distribute their galaxy's mass over a large area thereby producing a relatively shallow gravity well in the vicinity of the galaxy's disc. Sa galaxies on the other hand, have their arms more tightly wrapped around the galaxy's bulge, hence concentrating most of their mass in a much smaller volume and resulting in a deeper gravity well. Sb galaxies have properties intermediate between $\mathrm{Sa}$ and $\mathrm{Sc}$, but still their gravity wells would be deeper than those of Sc galaxies. Hence because Sa and Sb galaxies have deeper gravity wells, they would undergo the greater amount of photon blueshifting and hence should exhibit a smaller redshift than Sc galaxies, as is observed.

\section{Conclusions}

It has been demonstrated here that cosmological photons may progressively blueshift when traversing the gravity wells of galaxies and galaxy clusters. This 
effect is able to explain the blueshifting of galaxy spectra seen in large clusters such as the Virgo cluster as well as within smaller clusters such as the Local Group. The same blueshifting phenomenon is found to explain the Fingers-of-God effect as well as the Kaiser pancaking effect seen when galaxy cluster spectra are plotted in redshift space. It also explains why massive primary galaxies in a cluster are blueshifted relative to their companion galaxies. It also has been shown that excess tired-light redshifting of photons passing through cosmic voids can explain why voids appear elongated when plotted in redshift space. This variable Hubble constant effect also explains why galaxies residing in cosmic voids are dimmer and why those located in the centers of galaxy clusters are brighter than average.

The same photon energy behavior prediction also leads to a tired-light redshift relation which elsewhere has been shown to fit cosmological data better than the no evolution expanding universe cosmology [2] [3]. While other tired-light models have been advanced to explain only the cosmological redshift effect, only the gravity-potential-dependent photon energy change prediction of subquantum kinetics is able in addition to explain these photon blueshifting and excess photon redshifting phenomena. These various modes of photon energy behavior emerge as predictions of the equation system postulated to form the basis of subquantum kinetics, a methodology which to date has had at least 12 of its predictions verified [6] [7]. This gravity potential dependent variable Hubble "constant" prediction of subquantum kinetics satisfies Occam's razor in that it is able to explain a wide variety of cosmological phenomena which otherwise would require the introduction of numerous ad hoc assumptions for their explanation.

\section{Conflicts of Interest}

The author declares no conflicts of interest regarding the publication of this paper.

\section{References}

[1] Arp, H. (1987) Quasars, Redshifts, and Controversies. Cambridge University Press, Cambridge. https://doi.org/10.1017/CBO9780511564857

[2] LaViolette, P.A. (1986) Is the Universe Really Expanding? Astrophysical Journal, 301, 544-553. https://doi.org/10.1086/163922

[3] LaViolette, P.A. (2021) Expanding or Static Universe: Emergence of a New Paradigm. International Journal of Astronomy \& Astrophysics, in press.

[4] LaViolette, P.A. (1985) An Introduction to Subquantum Kinetics: I. An Overview of the Methodology. International Journal of General Systems, 11, 281-293. https://doi.org/10.1080/03081078508934918

[5] LaViolette, P.A. (1985) An Introduction to Subquantum Kinetics: II. An Open Systems Description of Particle and Fields. International Journal of General Systems, 11, 295-328. https://doi.org/10.1080/03081078508934919

[6] LaViolette, P.A. (1985) An Introduction to Subquantum Kinetics: III. The Cosmology of Subquantum Kinetics. International Journal of General Systems, 11, 329-345. 
https://doi.org/10.1080/03081078508934920

[7] LaViolette, P.A. (2012) Subquantum Kinetics: A Systems Approach to Physics and Cosmology. 4th Edition, Starlane Publications, Niskayuna.

[8] LaViolette, P.A. (2012) The Cosmic Ether: Introduction to Subquantum Kinetics. Physics Procedia, 38, 326-349. https://doi.org/10.1016/j.phpro.2012.08.032

[9] LaViolette, P.A. (2004) Genesis of the Cosmos. Bear \& Co., Rochester, VT.

[10] Yu-Lan, F. (1959) A Short History of Chinese Philosophy. Macmillan, New York, 695-697.

[11] Gmitro, J.I. and Scriven, L.E. (1966) A Physiochemical Basis for Pattern and Rhythm. In: Warren, K., Ed., Intracellular Transport, Academic Press, New York, 221-255. https://doi.org/10.1016/B978-1-4831-9872-9.50016-0

[12] Zwicky, F. (1929) On the Red Shift of Spectral Lines through Interstellar Space. Proceedings of the National Academy of Science, 15, 773-779. https://doi.org/10.1073/pnas.15.10.773

[13] Tammann, G.A. and Reindl, B. (2013) Alan Sandage and the Distance Scale. In: Proceedings of the International Astronomical Union, Volume 8, Symposium No. 289, 13-25. https://doi.org/10.1017/S1743921312021059

[14] Marosi, L.A. (2014) Hubble Diagram Test of 280 Supernovae Redshift Data. Journal of Modern Physics, 5, 29-33. https://doi.org/10.4236/jmp.2014.51005

[15] Marosi, L.A. (2019) Extended Hubble Diagram on the Basis of Gamma Ray Bursts Including the High Redshift Range of $\mathrm{z}=0.0331$ - 8.1. International Journal of Astronomy \& Astrophysics, 9, 1-11. https://doi.org/10.4236/ijaa.2019.91001

[16] LaViolette, P.A. (2021) The Origin of Gravity and Its Effects: According to the Subquantum Kinetics Paradigm. In: Krasnoholovets, V., Ed., The Origin of Gravity from the First Principles, Nova Science Publishers, Hauppauge, Chapter 7. (In Press)

[17] Mei, S., Blakeslee, J.P., Côté, P., et al. (2007) The ACS Virgo Cluster Survey. XIII. SBF Distance Catalog and the Three-Dimensional Structure of the Virgo Cluster. Astrophysical Journal, 655, 144-168. https://doi.org/10.1086/509598

[18] Tonry, J.L. (2000) The Surface Brightness Fluctuation Survey of Galaxy Distances. II. Local and Large-Scale Flows. Astrophysical Journal, 530, 625-651. https://doi.org/10.1086/308409

[19] Cappi, A. (1995) Gravitational Redshift in Galaxy Clusters. Astronomy \& Astrophysics, 301, 6-10.

[20] Stiavelli, M. and Setti, G. (1993) Nonequilibrium Motions in Galaxies and Gravitational Redshift. Monthly Notices of the Royal Astronomy Society, 262, L51-L54. https://doi.org/10.1093/mnras/262.1.L51

[21] Arp, H. (1994) Companion Galaxies: A Test of the Assumption That Velocities Can Be Inferred from Redshifts. Astrophysical Journal, 430, 74-82.

https://doi.org/10.1086/174383

[22] Kaiser, N. (1987) Clustering in Real Space and in Redshift Space. Monthly Notices of the Royal Astronomy Society, 227, 1-21. https://doi.org/10.1093/mnras/227.1.1

[23] Ryden, B.S. and Melott, A.L. (1996) Voids in Real Space and Redshift Space. Astrophysical Journal, 470, 160-171. https://doi.org/10.1086/177857

[24] Masatoshi, S. and Lee, J. (2012) Voids in Redshift Space. arXiv: 1203.0869.

[25] Ryden, B.S. (1995) Measuring $q_{0}$ from the Distortion of Voids in Redshift Space. Astrophysical Journal, 452, 25-32. https://doi.org/10.1086/176277 
[26] Hoyle, F., Rojas, R.R., Vogeley, M.S. and Brinkmann, J. (2005) The Luminosity Function of Void Galaxies in the Sloan Digital Sky Survey. Astrophysical Journal 620, 618-628. https://doi.org/10.1086/427176

[27] Murawski, W. (1983) An Intergalactic Absorbing Cloud in the Neighborhood of the North Galactic Pole. Acta Cosmologica, 12, 7-26.

[28] LaViolette, P.A. (1992) The Planetary-Stellar Mass-Luminosity Relation: Possible Evidence of Energy Nonconservation? Physics Essays, 5, 536-543.

[29] Tully, R.B., Shaya, E.J., Karachentsev, I.D., et al. (2008) Our Peculiar Motion Away from the Local Void. Astrophysical Journal, 676, 184-205.

https://doi.org/10.1086/527428

[30] Karachentsev, I.D., Kashibadze, O.G., Makarov, D.I. and Tully, R.B. (2009) The Hubble Flow around the Local Group. Monthly Notices of the Royal Astronomy Society, 393, 1265-1274. https://doi.org/10.1111/j.1365-2966.2008.14300.x

[31] Sandage, A. (1999) Bias Properties of Extragalactic Distance Indicators. VIII $H_{0}$ from Distance-Limited Luminosity Class and Morphological Type-Specific Luminosity Functions for SB, SBC, and SC Galaxies Calibrated Using Cepheids. Astrophysical Journal, 527, 479-487. https://doi.org/10.1086/308123

[32] Karachentsev, I.D., Chernin, A.D. and Teerikorpi, P. (2003) The Hubble Flow: Why Does the Cosmological Expansion Preserve Its Kinematical Identity from a Few Mpc Distance to the Observation Horizon? Astrophysics, 46, 399-414. https://doi.org/10.1023/B:ASYS.0000003255.06980.c0

[33] Arp, H. and Sulentic, J.W. (1985) Analysis of Groups of Galaxies with Accurate Redshifts. Astrophysical Journal, 291, 88-111. https://doi.org/10.1086/163044

[34] Arp, H. (1986) A Corrected Velocity for the Local Standard of Rest by Fitting to the Mean Redshift of Local Group Galaxies. Astronomy \& Astrophysics, 156, 207-212.

[35] Arp, H. (1982) Characteristics of Companion Galaxies. Astrophysical Journal, 256, 54-74. https://doi.org/10.1086/159883

[36] Bottinelli, L. and Gouguenheim, L. (1973) Redshifts of Companion Galaxies. Astronomy \& Astrophysics, 26, 85-89.

[37] Arp, H.T. (1990) The Hubble Relation: Differences between Galaxy Types Sb and Sc. Astrophysics \& Space Science, 167, 183-219. https://doi.org/10.1007/BF00659347

[38] Russell, D.G. (2005) Evidence for Intrinsic Redshifts in Normal Spiral Galaxies. $A s$ trophysics \& Space Science 298, 577-602.

https://doi.org/10.1007/s10509-005-2317-x 